\title{
Temas pendientes en la salud infantil
}

\author{
Pending issues in child health
}

En el año 2000 los gobernantes del mundo asumieron el compromiso de "no escatimar esfuerzos para liberar a nuestros semejantes, hombres, mujeres y niños de las condiciones abyectas y deshumanizadoras de la pobreza extrema", este compromiso fue plasmado en un marco de trabajo inspirador de ocho objetivos, y después en pasos prácticos de amplio espectro que han permitido a personas en todo el mundo mejorar sus vidas y sus perspectivas de futuro.El cuarto objetivo: reducir la mortalidad de los niños menores de 5 años, expresa la preocupación por la salud infantil como una prioridad a nivel mundial (1).

El informe del 2015 de los Objetivos de Desarrollo del Milenio revela que la tasa mundial de mortalidad de niños menores de 5 años ha disminuido en más de la mitad, reduciéndose de 90 a 43 muertes por cada 1000 niños nacidos vivos entre 1990 y 2015. En nuestro país en 1990 de cada 1000 niños menores de 5 años morían 79. En el 2012 morían 18 de cada mil nacidos vivos. El informe del Estado Mundial de la Infancia 2014 señala que, en nuestro país en gran medida, la disminución de la tasa de mortalidad infantil se debe a los progresos en la prestación de servicios de inmunización, salud y agua y saneamiento (2).

El Ministerio de Salud del Perú por su parte ha desarrollado acciones a nivel político como: promover el compromiso intersectorial e intergubernamental para promover el desarrollo infantil temprano, formulación de lineamientosy diversos planes dirigidos a abordar problemas de salud infantil como desnutrición crónica, prevención de anemia entre otros, así como para la reducción de la mortalidad y morbilidad neonatal y prevención del embarazo en adolescentes.Así también se han desarrollado acciones para Incrementar el acceso a los servicios de salud: extensión de la cobertura de acceso al Seguro Integral de Salud (SIS) a las gestantes y niños menores de 5 años, priorizando a los menores de 3 años, universalizar de manera progresiva la suplementación con hierro y otros micronutrientes a niños menores de 3 años. Finalmente se reconoce el desarrollo de estrategias educativas y comunicacionales para mejorar conocimientos, actitudes y prácticas de la mujer, familia y comunidad para el cuidado de la mujer en edad fértil, gestantes, puérperas y de los recién nacidos (3).

Cabe resaltar que a pesar de todas las acciones desarrolladas y los logros alcanzados respecto a la salud infantil, en nuestro país,aún existen temas pendientes de atención por parte de los servicios, equipos y profesionales de salud respecto a la morbilidad infantil debido a los cambios en el perfil epidemiológico como: el incremento de malformaciones congénitas deformaciones y anomalías cromosómicas, traumatismos, envenenamientos, patologías que implican el abordaje especializado e integral del paciente con intervenciones dirigidas a los cuidadores y familia para cubrir las necesidades físicas, emocionales, psicológicas y espirituales desde el momento del diagnóstico hasta el final de la vida, tanto en los periodos de internamiento como para el cuidado en el hogar. Necesidades y/o problemas que muchas veces no son atendidos y que impactan directamente en la calidad de vida, así como en el afrontamiento familiar. 
En la presente edición los artículos abordan diversos problemas o situaciones en los que el profesional de enfermería tiene un rol importante como eje de articulación del cuidado, integrando la participación efectiva de los miembros del equipo de salud para atender las necesidades del paciente y familia.

\section{Carolina Cerna Silva ${ }^{1, a}$}

\section{REFERENCIAS BIBLIOGRAFICAS}

1. Naciones Unidas. Objetivos de Desarrollo del Milenio Informe de 2015. New York: Naciones Unidas; 2015. (Citado junio del 2016) Disponible en: http://www.un.org/es/millenniumgoals/pdf/2015/mdg-report-2015 spanish.pdf

2. Fondo de las Naciones Unidas para la Infancia. El estado mundial de la infancia de 2014 en cifras. Todos los niños cuentan. New York:.UNICEF; 2014. (Citado junio del 2016) Disponible en: http://www.unicef. org/peru/spanish/estado-mundial-infancia-2014-unicef.pdf

3. Ministerio de Salud. Situación de Salud y Nutrición Materno Infantil en el Perú. Lima, Perú: Ministerio de Salud; 2014. (Citado junio del 2016) Disponible en: http://www.paho.org/nutricionydesarrollo/wp-content/ uploads/2014/04/Lucia-Solis-Situacion-de-Salud-y-Nutricion-Materno-Infantil-en-el-Peru.pdf

1 Facultad de Enfermería, Universidad Peruana Cayetano Heredia. Lima, Perú.

a Licenciada en Enfermería, con Estudios de Especialidad en Enfermería Pediátrica. 